\title{
Nonlinear evolution of the modulational instability under weak forcing and damping
}

\author{
J. Touboul ${ }^{1}$ and C. Kharif ${ }^{2}$ \\ ${ }^{1}$ Laboratoire de Sondages Éléctromagnétiques de l'Environnement Terrestre, UMR 6017, Institut des Sciences de l'Ingénieur \\ Toulon - Var, Av. G. Pompidou, BP 56, 83162, La Valette Cedex, France \\ ${ }^{2}$ Institut de Recherche sur les Phénomènes Hors Équilibre, UMR 6594, École Centrale Marseille, \\ Technopôle de Château-Gombert, 49 rue Joliot Curie - BP 146, 13384, Marseille Cedex 13, France
}

Received: 10 September 2010 - Revised: 14 November 2010 - Accepted: 16 November 2010 - Published: 17 December 2010

\begin{abstract}
The evolution of modulational instability, or Benjamin-Feir instability is investigated within the framework of the two-dimensional fully nonlinear potential equations, modified to include wind forcing and viscous dissipation. The wind model corresponds to the Miles' theory. The introduction of dissipation in the equations is briefly discussed. Evolution of this instability in the presence of damping was considered by Segur et al. (2005a) and Wu et al. (2006). Their results were extended theoretically by Kharif et al. (2010) who considered wind forcing and viscous dissipation within the framework of a forced and damped nonlinear Schrödinger equation. The marginal stability curve derived from the fully nonlinear numerical simulations coincides with the curve obtained by Kharif et al. (2010) from a linear stability analysis. Furthermore, it is found that the presence of wind forcing promotes the occurrence of a permanent frequency-downshifting without invoking damping due to breaking wave phenomenon.
\end{abstract}

\section{Introduction}

Since Stokes (1847), fully nonlinear potential water wave equations are known to admit two dimensional uniform propagative solutions, the so called Stokes' waves. However, pioneer works of Lighthill (1965) provided a geometric condition for the stability of these waves. Later on, Benjamin and Feir (1967) showed analytically that Stokes' waves of moderate amplitude are unstable to long wave perturbations of small amplitude travelling in the same

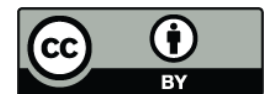

Correspondence to: J. Touboul (julien.touboul@univ-tln.fr) direction. This instability is named the Benjamin-Feir instability (or modulational instability). This result was derived independently by Whitham (1974) in an averaged Lagrangian approach and by Zakharov (1968) who used a Hamiltonian formulation of the water wave problem. Using this approach, the latter author derived the nonlinear Schrödinger equation (NLS), and confirmed the previous stability results.

Within the last fifty years, the study of this instability became central for fundamental and applied research. The modulation instability is one of the most important mechanisms for the formation of rogue waves (Kharif and Pelinovsky, 2003). A complete review on the various phenomena yielding to rogue waves can be found in the book of Kharif et al. (2009). In the absence of forcing and damping, Stokes' waves of specific initial steepness are submitted to this instability, when they encounter perturbations of specific wave numbers (Dold and Peregrine, 1986; Banner and Tian, 1998). In this case, they encounter a nonlinear quasi-recursive evolution, the so called FermiPasta-Ulam recurrence phenomenon (Fermi et al., 1955). This phenomenon corresponds to a series of modulationdemodulation cycles, during which initially uniform wave trains become modulated, possibly leading to the formation of a huge wave. Modulation is due to an energy transfer from the wave carrier to the unstable sidebands. In the wave number space, these unstable sidebands are located in a finite narrow band centred around the carrier wave number. During the demodulation, the energy returns to the fundamental component of the original wave train. Using the Zakharov equation, Shemer (2010) questions the relevance of the Benjamin-Feir index based on the linear modulational stability analysis, to indicate the intensity of nonlinear interactions of a carrier wave with finite amplitude sidebands.

Published by Copernicus Publications on behalf of the European Geosciences Union. 
More precisely, he emphasized that nonlinear interactions also occur for sidebands located beyond the Benjamin-Feir instability domain.

A damped nonlinear Schrödinger equation (dNLS) was derived by Joo et al. (1991) who revisited the BenjaminFeir instability in the presence of dissipation. They studied numerically the evolution of narrow bandwidth waves of moderate amplitude. More recently, Segur et al. (2005a) theoretically investigated the modulational instability within the framework of the dNLS equation and demonstrated that any amount of dissipation stabilizes the modulational instability in the sense of Lyapunov. Namely, they showed that the zone of the unstable region, in the wavenumber space, shrinks as time increases. As a result, any initially unstable mode of perturbation will finally become stable. Segur et al. (2005a) have confirmed their theoretical predictions by laboratory experiments for waves of small to moderate amplitude. Later, Wu et al. (2006) developed fully nonlinear numerical simulations which agreed with the theory and experiments of Segur et al. (2005a).

From the latter study, we could conclude that dissipation may prevent the development of the Benjamin-Feir instability. This effect questions the occurrence of modulational instability of water wave trains in the field. Segur et al. (2005b) speculated about the effect of dissipation on the early development of rogue waves and raised the question whether or not the Benjamin-Feir instability was able to spawn a rogue wave.

Nevertheless, these authors did not take the effect of wind into account. When considering the occurrence of modulational instability in the field, the role of wind upon this instability in the presence of dissipation needs to be addressed. Based on this assumption, Kharif et al. (2010) derived a forced and damped nonlinear Schrödinger equation (fdNLS) and extended the analysis of Segur et al. (2005a) when wind input is introduced. The influence of wind was introduced through a pressure term acting at the interface, in phase with the wave slope, accordingly to Miles' theory (Miles, 1957). This quasi-laminar theory of wind wave amplification is based on the Miles' shear flow instability. This mechanism of wave amplification is a resonant interaction between water waves and a plane shear flow in air which occurs at the critical height where the wind velocity matches the phase velocity of the surface waves. Stokes waves propagating in the presence of such a forcing, when not submitted to modulational instability, encounter an exponential growth. They demonstrated, within the framework of dfNLS equation, that Stokes' waves were unstable to modulational instability as soon as the friction velocity is larger than a threshold value. Conversely, for a given friction velocity it was found that only carrier waves presenting frequencies (or wavenumbers) lower than a threshold value are subject to Benjamin-Feir instability. Otherwise, due to dissipation, modulational instability restabilizes in the sense of Lyapunov.
In a previous work, Kharif and Touboul (2010) investigated the development of the modulational instability under wind action and viscous dissipation within the framework of fully nonlinear potential equations. This work is an extension of that of Wu et al. (2006) when wind input is considered. It extends the result of Kharif et al. (2010) to higher orders of nonlinearity and larger band spectra, too. The marginal stability diagram obtained theoretically by Kharif et al. (2010) was globally reproduced, with slight differences observed for the youngest waves. This disagreement was correlated to the way in which the viscosity is introduced in the fully nonlinear equations.

In Sect. 2, we remind the main results obtained by Kharif et al. (2010). Section 3 is devoted to a brief presentation of the governing water wave equations and the numerical method. Results of the numerical simulations and comparison with those of Kharif et al. (2010) are given in Sect. 4 and a conclusion is provided in Sect. 5.

\section{The forced and damped NLS equation}

Recently, Kharif et al. (2010) used a forced and damped version of the nonlinear Schrödinger equation

$$
\begin{gathered}
i\left(\psi_{t}+C_{\mathrm{g}} \psi_{x}\right)-\frac{\omega_{0}}{8 k_{0}^{2}} \psi_{\mathrm{xx}}-2 \omega_{0} k_{0}^{2}|\psi|^{2} \psi \\
=i \frac{\mathcal{W} \omega_{0} k_{0}}{2 g \rho} \psi-2 i \nu k_{0}^{2} \psi
\end{gathered}
$$

to investigate both damping and amplification effects on the Benjamin-Feir instability. Herein, $\mathcal{W}=\rho_{\mathrm{a}} \beta u_{*}^{2} / \kappa^{2}$ represents the wind effect and $v$ water viscosity. Parameters $k_{0}$ and $\omega_{0}$ are the wavenumber and frequency of the carrier wave, satisfying the linear dispersion relation $\omega_{0}^{2}=g k_{0}, g$ is the gravity, $C_{\mathrm{g}}=\omega_{0} / 2 k_{0}$ is the group velocity of the carrier wave, $\rho$ and $\rho_{\mathrm{a}}$ are the water and air densities, respectively, $\kappa$ is the von Karman constant, $u_{*}$ is the friction velocity of the wind and $\beta$ is the Miles' coefficient depending on the carrier wave age. Equation (1) describes the spatial and temporal evolution of the envelope, $\psi$, of the surface elevation, $\eta$, of weakly nonlinear and dispersive gravity waves on deep water when dissipation, due to viscosity, and amplification, due to wind, are considered.

Kharif et al. (2010) found that the stability of the envelope depends on the frequency of the carrier wave and the friction velocity of the wind over the waves and plotted the critical curve separating stable envelopes from unstable envelopes. Namely, they showed that for a given friction velocity $u_{*}$, only carrier wave of frequency $\omega_{0}$ which satisfies the following condition are unstable to modulational perturbations

$$
\frac{4 \nu \kappa^{2} \omega_{0}}{\beta\left(\kappa c_{0} / u_{*}\right) s u_{*}^{2}}<1 .
$$


This condition can be rewritten as follows

$\frac{\mathcal{A}^{2} \Omega}{\beta(\mathcal{A})}<1$

where $\mathcal{A}=\kappa c_{0} / u_{*}$ is associated to wind, whereas $\Omega=$ $\omega_{0} /\left(s g^{2} / 4 v\right)^{1 / 3}$ is associated to dissipation. The parameters $c_{0}=\omega_{0} / k_{0}$ and $s=\rho_{\mathrm{a}} / \rho$ are the phase velocity of the carrier wave and density ratio. Note that $\mathcal{A}^{2} \Omega / \beta(\mathcal{A})$ is equal to the ratio between the rate of damping and rate of amplification and illustrates the competition between dissipative effects and wind input. The non-dimensional numbers $\mathcal{A}$ and $\Omega$ correspond to the wave age and non-dimensional carrier wave frequency.

The modulational instability was found to be sustained as soon as the friction velocity is larger than a threshold value. Conversely, for a given friction velocity, it was found that only carrier waves presenting frequencies (or wavenumbers) lower than a threshold value are subject to Benjamin-Feir instability. Otherwise, due to dissipation, modulational instability restabilizes in the sense of Lyapunov.

\section{The fully nonlinear approach and numerical method}

\subsection{Governing equations}

The numerical approach used in this study is based on the High-Order Spectral Method (HOSM), simultaneously developed by Dommermuth and Yue (1987) and West et al. (1987). This method assumes that the flow is potential. However, non-potential effects due to wind and viscosity can be taken into account through a modification of the boundary conditions at the surface.

The wind has already been introduced in the dynamic boundary condition through a pressure term acting at the free surface in several numerical potential models. Among them, one may cite Touboul et al. (2006) and Touboul et al. (2008) who introduced and discussed this approach for BIEM methods and Touboul and Kharif (2006) and Touboul (2007) who extended it to HOS methods. The pressure term used here is based on the Miles' theory (Miles, 1957), accordingly to the approach of Kharif et al. (2010).

The viscosity was introduced heuristically by $\mathrm{Wu}$ et al. (2006) who used the HOS method to address the question raised in Segur et al. (2005a) on the restabilisation of the Benjamin-Feir instability of a Stokes wave train in the presence of dissipation. The introduction was made through the addition of a damping term in the dynamic boundary condition. In our previous work (Kharif and Touboul, 2010), we used the same formalism. The system of equations considered was

$$
\begin{aligned}
& \phi_{\mathrm{xx}}+\phi_{\mathrm{zz}}=0 \text { for }-\infty<\mathrm{z}<\eta(\mathrm{x}, t) \\
& \nabla \phi \rightarrow 0 \text { for } \mathrm{z} \rightarrow-\infty \\
& \eta_{t}+\phi_{\mathrm{x}} \eta_{\mathrm{x}}-\phi_{\mathrm{z}}=0 \text { for } \mathrm{z}=\eta
\end{aligned}
$$

$$
\begin{aligned}
& \phi_{t}+\frac{1}{2}\left[\left(\phi_{\mathrm{x}}\right)^{2}+\left(\phi_{\mathrm{z}}\right)^{2}\right]+g \eta= \\
&-\frac{P_{\mathrm{a}}}{\rho}-2 v \phi_{\mathrm{zz}} \text { for } \mathrm{z}=\eta
\end{aligned}
$$

where $\phi$ is the velocity potential, $\eta$ is the surface elevation, $t$ is the time, $\mathrm{x}$ and $\mathrm{z}$ are the horizontal and vertical coordinates, respectively, $g$ is the gravity, $P_{\mathrm{a}}$ is the pressure at the surface elevation, $\rho$ is the water density and $v$ is the kinematic viscosity. Wind effect is introduced through $P_{\mathrm{a}}$.

Following Ruvinsky et al. (1991) and considering the frequency-downshifting phenomenon (Skandrani et al., 1996) introduced a damping term correlated to the vorticity generated by viscosity in a thin layer near the water surface. As a result, both kinematic and dynamic boundary conditions were modified and an additional boundary condition was derived describing the rate of change of the vortical component at the surface. However, another approach was derived properly by Lundgren (1989) within the framework of linearized equations through the modification of both kinematic and dynamic boundary conditions, but avoiding the use of the third boundary condition introduced by Skandrani et al. (1996). Equivalence between the previous formalisms was demonstrated by Longuet-Higgins (1992). Recently, Dias et al. (2008) confirmed this result within the framework of the linear theory and suggested extending it to the case of the fully nonlinear set of equations. Joo et al. (1991) derived a damped version of the NLS equation, demonstrating that the linear damping term was correct up to the third-order. The kinematic (6) and dynamic (7) boundary conditions now read

$$
\begin{aligned}
\eta_{t}+\phi_{\mathrm{x}} \eta_{\mathrm{x}}-\phi_{\mathrm{z}}-2 v \eta_{\mathrm{xx}}=0 & \text { for } \mathrm{z}=\eta \\
\phi_{t}+\frac{1}{2}\left[\left(\phi_{\mathrm{x}}\right)^{2}+\left(\phi_{\mathrm{z}}\right)^{2}\right]+g \eta & = \\
-\frac{P_{\mathrm{a}}}{\rho}-2 v \phi_{\mathrm{zz}} & \text { for } \mathrm{z}=\eta,
\end{aligned}
$$

while Eqs. (4)-(5) remain unchanged. The differences between the sets of Eqs. (6)-(7) and (8)-(9) are weak, but they are responsible for a significant change in the kinematic and dynamics of the water waves. In the absence of wind $\left(P_{\mathrm{a}}=0\right)$, the linearized version of Eqs. (4)-(7) admits a dispersion relationship which reads

$$
\left(\omega_{0}+i v k_{0}^{2}\right)^{2}=g k_{0}-v^{2} k_{0}^{4},
$$

whereas the linearization of the system of Eqs. (4), (5), (8), (9) yields to the following dispersion relationship

$$
\left(\omega_{0}+2 i v k_{0}^{2}\right)^{2}=g k_{0}
$$

It is clear that relation (10) corresponds to water waves propagating with a phase velocity $c=\sqrt{g / k_{0}-v^{2} k_{0}^{2}}$ and damped with a characteristic time scale $\tau=1 / \nu k_{0}^{2}$, whereas 
the relation (11) corresponds to waves propagating with a phase velocity $c=\sqrt{g / k_{0}}$, and damped with a characteristic time scale $\tau=1 / 2 v k_{0}^{2}$. As a result, the first set of equations corresponds to a modification of the physics of Stokes' waves and predicts a damping rate twice as low as usual (Lamb, 1932).

Once we know the proper set of equations, we follow the classical decomposition of Dommermuth and Yue (1987) and we introduce the velocity potential at the free surface $\phi^{\mathrm{s}}(x, t)=\phi(x, \eta(x, t), t)$. The kinematic and dynamic free surface conditions (8) and (9), once made non-dimensional, now write

$$
\begin{gathered}
\eta_{t}=-\phi_{\mathrm{x}}^{\mathrm{s}} \eta_{\mathrm{x}}+W\left(1+\eta_{\mathrm{x}}^{2}\right)+2 \frac{\nu k_{0}}{c_{0}} \eta_{\mathrm{xx}} \\
\phi_{t}^{\mathrm{s}}=-\eta-\frac{1}{2} \phi_{\mathrm{x}}^{s^{2}}+\frac{1}{2} W^{2}\left(1+\eta_{x}^{2}\right)+2 \frac{\nu k_{0}}{c_{0}} W \eta_{\mathrm{xx}} \\
+2 \frac{\nu k_{0}}{c_{0}} \frac{\phi_{\mathrm{xx}}^{\mathrm{s}}-W \eta_{\mathrm{xx}}}{1+\eta_{\mathrm{x}}^{2}}-p_{\mathrm{a}}
\end{gathered}
$$

where $p_{\mathrm{a}}=P_{\mathrm{a}} / \rho c_{0}^{2}=s \beta\left(u_{*} / \kappa c_{0}\right)^{2} \eta_{\mathrm{x}}$ is the pressure term corresponding to Miles' theory. Note that the coefficient $\beta$ depends on the phase velocity of the carrier $c_{0}$ and on the friction velocity $u_{*}$ (see Conte and Miles, 1959). The group $v k_{0} / c_{0}$ plays the role of the inverse of a Reynolds number.

\subsection{Initial conditions}

From a numerical point of view, one part of the initial condition is obtained by considering a Stokes wavetrain $(\bar{\eta}$, $\bar{\phi}$ ) which is computed using the approach first introduced by Longuet-Higgins (1985). A very high-order Stokes wave of amplitude $a_{0}$ and wavenumber $k_{0}$ is calculated iteratively. In the absence of wind and dissipation, the infinitesimal perturbation components $\left(\eta^{\prime}, \phi^{\prime}\right)$ calculated through a perturbative approach developed by Kharif and Ramamonjiarisoa (1988) correspond to a Benjamin-Feir instability of wavenumber $\delta k$. The perturbed Stokes wave is obtained by adding the infinitesimal perturbations at the sidebands $k_{0} \pm \delta k$ of the fundamental and its harmonics. For fixed values of $(\mathcal{A}, \Omega)$ two kinds of initial conditions are used when wind and dissipation are considered. The first kind (unseeded case) corresponds to the unperturbed Stokes' wave $(\eta, \phi)=(\bar{\eta}, \bar{\phi})$, whereas the second kind (seeded case) corresponds to the perturbed Stokes' wave $(\eta, \phi)=(\bar{\eta}, \bar{\phi})+$ $\varepsilon\left(\eta^{\prime}, \phi^{\prime}+\bar{\phi}_{\mathrm{Z}} \eta^{\prime}\right)$, with $\varepsilon=10^{-3}$. In both cases, we consider a Stokes wavetrain such as $a_{0} k_{0}=0.11$ and $k_{0}=5$. The wavenumber of the modulational instability is $\delta k=1$. This choice of the perturbation wave number corresponds to the closest approximation of the most unstable wave number that can be fitted in the computational domain. The order of nonlinearity was taken equal to $M=6$. In other words, nonlinear terms have been retained up to the sixth-order. The highest wavenumber taken into account in the simulations is $k_{\max }=50$, corresponding to the ninth harmonic of the fundamental wavenumber. The number of mesh points was taken equal to $N=750$, satisfying the stability criterion $N>(M+1) \cdot k_{\max }$. In the absence of wind and damping, the unperturbed initial condition leads to the steady evolution of the Stokes' wavetrain, whereas the perturbed initial condition leads to the well-known Fermi-Pasta-Ulam recurrence. We propagate these initial wavetrains under various conditions of wind and dissipation, to analyse the behaviour of the modulational instability of the Stokes wavetrain.

\section{Results and comparison}

Since Stokes' waves are propagating under the action of wind and viscosity, this flow cannot be considered stationary nor periodic, leading the definition of instability to be unclear. To discuss the combined influence of wind forcing and damping on the modulational instability, we need to define a reference flow. Thus, we first consider the evolution of the unperturbed Stokes' waves in the presence of forcing and dissipation (unseeded case). Afterwards, the evolution of the initially perturbed Stokes' wave train is considered under the same conditions of wind forcing and damping (seeded case). The nonlinear evolution of the Stokes' wavetrain perturbed by the modulational instability in the presence of wind and dissipation is then compared to that of the reference flow. In that way, the deviation from the reference flow can be interpreted in terms of modulational instability and the influence of wind forcing and dissipation can be analysed. This approach, introduced in our previous work (Kharif and Touboul, 2010), is modified here to introduce the energy of the perturbation.

In the Figs. 1 and 2 are plotted the time evolution of the amplitudes of the fundamental mode $k=5$ and sidebands $k=$ 4 and $k=6$ for two kinds of initial conditions corresponding to unseeded and seeded cases. The figures correspond to two different conditions of wind forcing and damping.

Figure 1 shows the time evolution of the normalized amplitudes $a(t) / a_{0}$ of the fundamental mode $k=5$, subharmonic mode $k=4$ and superharmonic mode $k=$ 6 with and without modulational instability. For both cases, the simulations correspond to a wind parameter $\mathcal{A}=4$ and to a viscosity parameter $\Omega=0.59$. Within the framework of the NLS equation, Kharif et al. (2010) showed that the wave train is unstable to modulational instability for these values of $\mathcal{A}$ and $\Omega$. From this figure, it appears that both wavetrains (unseeded and seeded cases) present a similar evolution during the first hundred periods of propagation, $T$ being the fundamental wave period. Then, the behaviour of the wavetrain is strongly affected by the development of the modulational instability. For the unperturbed case (unseeded case), the fundamental component increases, since no occurrence of the modulational instability is expected. However, due to 


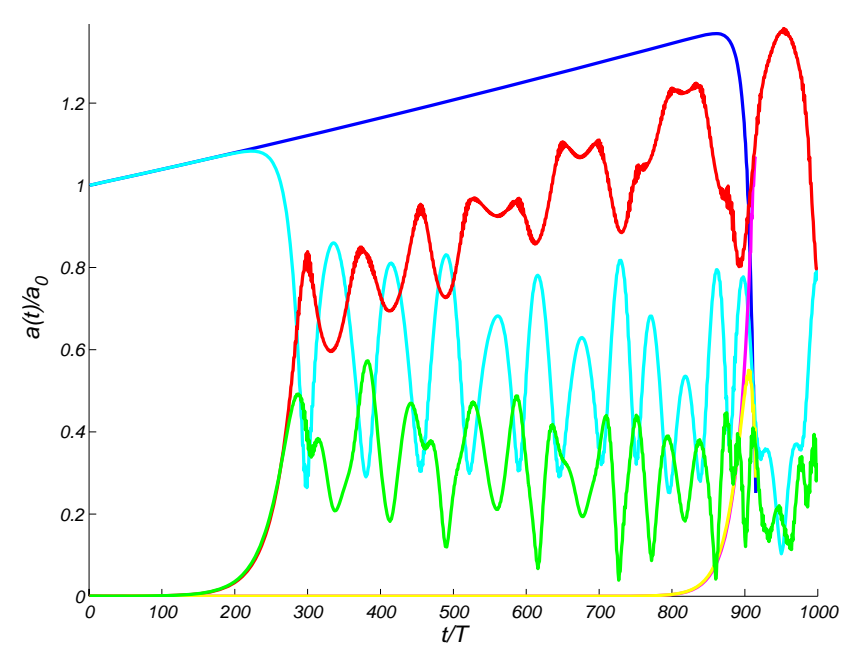

Fig. 1. Time evolution of the normalized amplitudes of the fundamental mode $(k=5)$, subharmonic mode $(k=4)$ and superharmonic mode $(k=6)$ for $(\mathcal{A}, \Omega)=(4,0.59)$. Fundamental mode amplitude (-), subharmonic mode amplitude (-) and superharmonic mode amplitude $(-)$ for an initially unperturbed Stokes' wave (unseeded case). Fundamental mode amplitude (-), subharmonic mode amplitude (-) and superharmonic mode amplitude (-) for an initially perturbed Stokes' wave (seeded case). $T$ is the fundamental wave period.

the accumulation of numerical errors, the spontaneous occurrence of the modulational instability cannot be avoided, but not before $t=900 T$. For the initially perturbed case (seeded case), the development of the modulational instability is responsible for the frequency downshift observed at around $t=500 T$, already discussed by Skandrani et al. (1996) in the presence of only molecular viscosity and by Hara and Mei (1991) in the presence of wind and eddy viscosity. One can see that the subharmonic component increases continuously whereas the fundamental and superharmonic component decreases. The superharmonic component decreases faster than the fundamental component. Hence, wind energy goes to the subharmonic mode, whereas dissipation reduces the fundamental and superharmonic components. During the modulation process, a broadening of the spectrum is observed, even if not presented here for the sake of clarity.

On Fig. 3 one can observe the persistence of the modulational instability through the evolution of the free surface. Beyond $T=500 T$ the dominant mode is the subharmonic sideband corresponding to a permanent frequency-downshifting. This result is obtained without invoking dissipation due to breaking wave phenomenon as shown by Trulsen and Dysthe (1990) within the framework of gravity waves. However, it has to be mentioned that these authors predicted a delay in the modulational instability in the presence of strong winds and eventually its disappearance

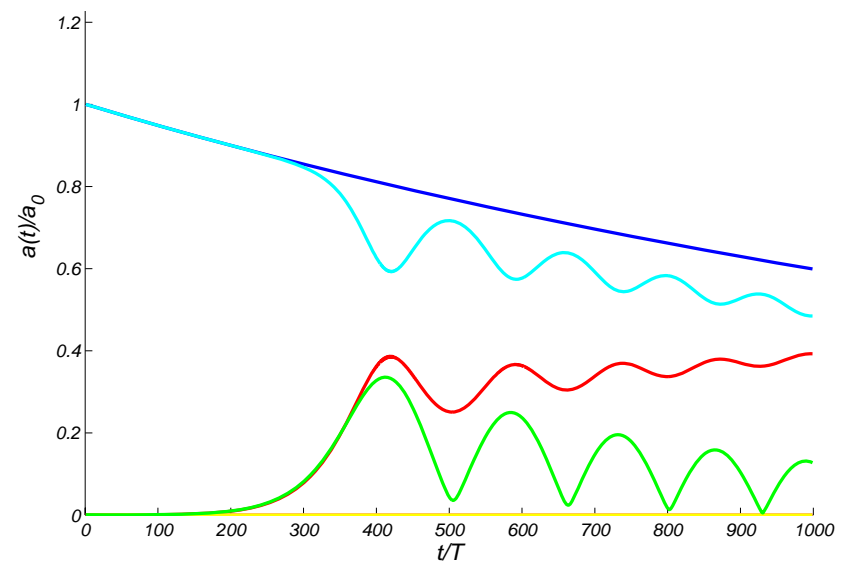

Fig. 2. Time evolution of the normalized amplitudes of the fundamental mode $(k=5)$, subharmonic mode $(k=4)$ and superharmonic mode $(k=6)$ for $(\mathcal{A}, \Omega)=(4,0.61)$. Fundamental mode amplitude (-), subharmonic mode amplitude (-) and superharmonic mode amplitude (-) for an initially unperturbed Stokes' wave (unseeded case). Fundamental mode amplitude (-), subharmonic mode amplitude (-) and superharmonic mode amplitude (-) for an initially perturbed Stokes' wave (seeded case). $T$ is the fundamental wave period.

for the strongest winds, using an approach based on a higherorder nonlinear Schrödinger equation (Trulsen and Dysthe, 1992).

Figure 2 corresponds to $(\mathcal{A}, \Omega)=(4,0.61)$. The wind condition is similar to the previous numerical simulation, but the dissipative effect considered is stronger. This case corresponds to a linearly stable case of the modulational instability, as obtained by Kharif et al. (2010) in the framework of the NLS equation. From this figure, one can see that wind energy goes to the subharmonic mode, whereas dissipation reduces the fundamental and superharmonic components, as previously observed. However, modulation of modes decrease and they present a monotonic behaviour. For the unseeded case, as expected, we observe an exponential decay of the fundamental mode. Note that there is no natural occurrence of the subharmonic mode of the modulational instability as it was found in Fig. 1. For the seeded case, the first maximum modulation that occurs at $t=410 T$ is followed by partial damped modulation/demodulation cycles. Figure 4 illustrates the disappearance of the modulational instability through the evolution of the free surface. In this case, dissipation prevails over amplification due to wind and Segur et al. (2005a) have obtained linear and nonlinear stability of modulational perturbations within the framework of the dissipative NLS equation. More specifically they showed that dissipation reduces the set of unstable wavenumbers as time increases. 

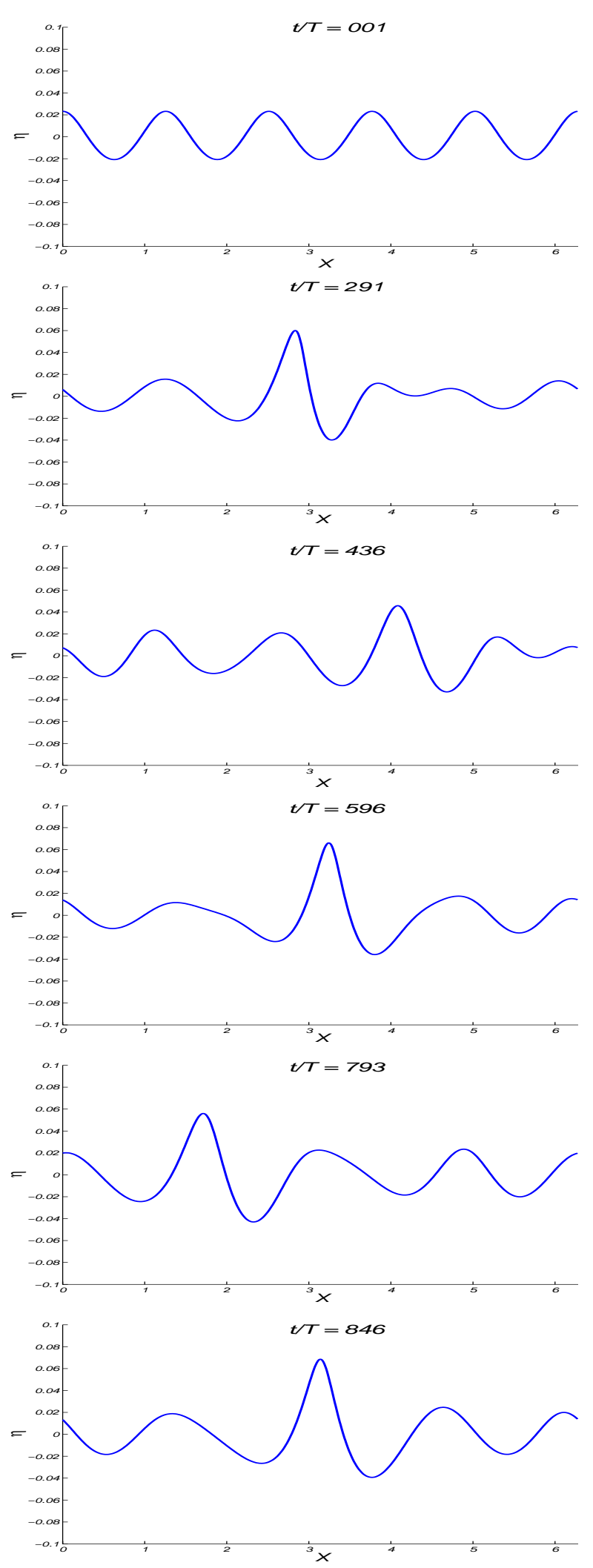

Fig. 3. Surface wave profiles at different times, obtained while propagating initial condition corresponding to seeded case with $(\mathcal{A}, \Omega)=(4,0.59)$. From top to bottom $t / T=1,291,436,596$, 793,846 .
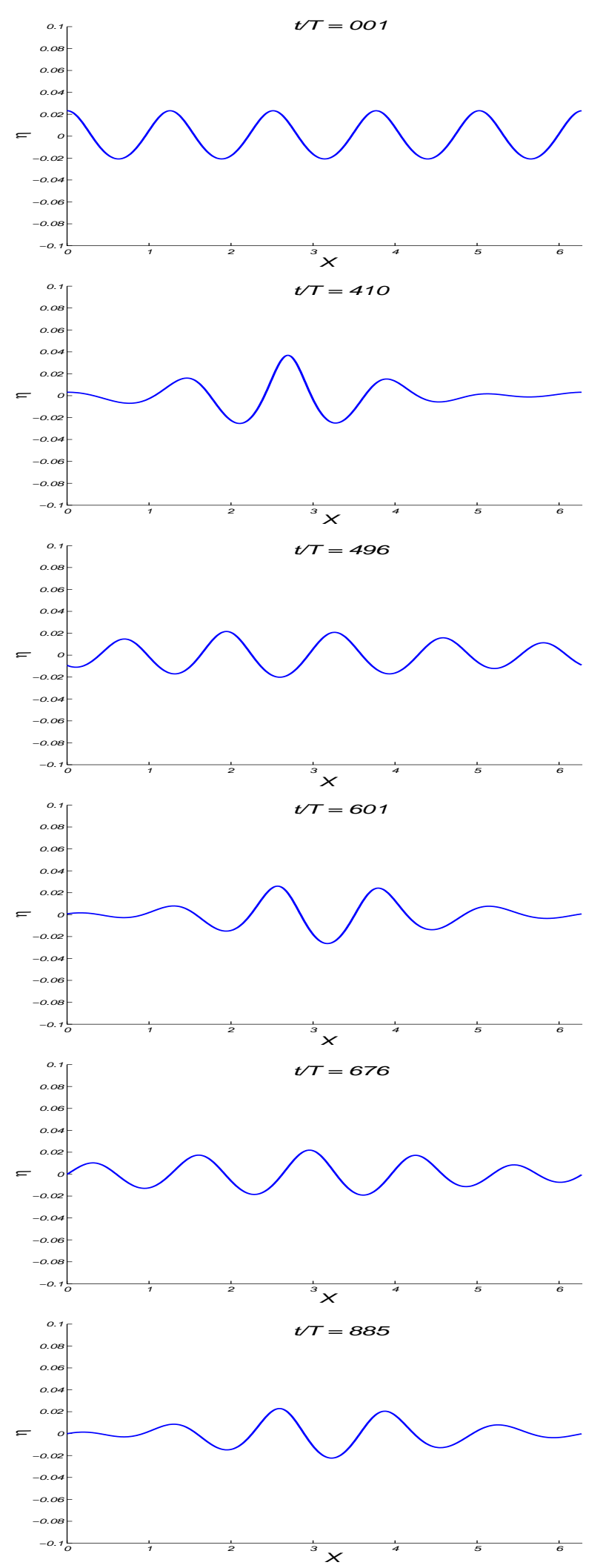

Fig. 4. Surface wave profiles at different times, obtained while propagating initial condition corresponding to seeded case with $(\mathcal{A}, \Omega)=(4,0.61)$. From top to bottom $t / T=1,410,496,601$, 676,885 . 


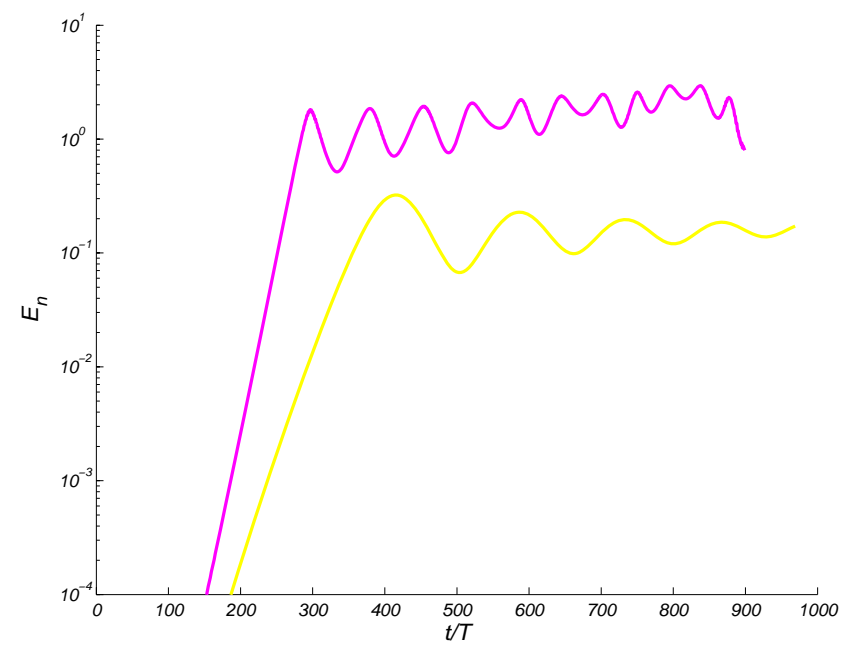

Fig. 5. Time evolution of the norm $E_{\mathrm{n}}$ for $(\mathcal{A}, \Omega)=(4,0.59)(-)$ and $(\mathcal{A}, \Omega)=(4,0.61)(-)$.

Consequently every mode becomes stable. The result of this numerical simulation agrees with that of Segur et al. (2005a) and $\mathrm{Wu}$ et al. (2006) who considered only dissipation. In their approach, a solution is said to be stable if every solution that starts close to this solution at $t=0$ remains close to it for all $t>0$, otherwise the solution is unstable. To include a nonlinear stability analysis they introduced a norm and considered the stability as that from Lyapunov.

In our previous work, we assumed that the dominant mode describes the main behaviour of a wave train and we introduced a norm measuring the distance between the fundamental modes of the unperturbed and perturbed Stokes wave corresponding to the unseeded case and seeded case, respectively. However, it is more consistent to consider the energy of the perturbation. Thus, a new norm can be introduced as

$E_{n}(t)=\frac{\int_{-\infty}^{\infty}\left(a_{k_{\mathrm{S}}}(t)-a_{k_{\mathrm{US}}}(t)\right)^{2} d k}{\int_{-\infty}^{\infty} a_{k_{\mathrm{US}}}^{2}(0) d k}$,

where $a_{k_{\mathrm{US}}}(t)$ is the amplitude of the water component elevation $\eta$ of wave number $k$, for the initially unperturbed wave train (unseeded case) and $a_{k_{\mathrm{S}}}(t)$ is the amplitude of the component of water elevation $\eta$ of wave number $k$, for the initially perturbed wave train (seeded case). This norm corresponds to the potential energy of the perturbation. Its value characterises the deviation of the perturbed solution from the unperturbed solution.

Figure 5 shows the time evolution of this norm for two sets of parameters $(\mathcal{A}, \Omega)=(4,0.59)$ and $(\mathcal{A}, \Omega)=$ $(4,0.61)$. For the two cases, we can observe two regimes. The first regime corresponds with the development of the modulational instability and shows that it is the nonlinear

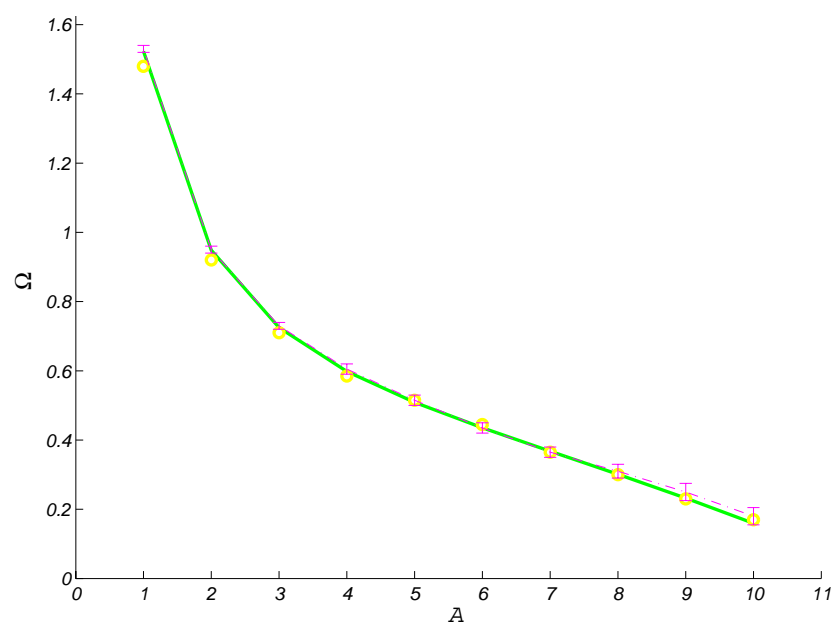

Fig. 6. Theoretical (-) and numerical (- - -) marginal stability contour lines. ( $)$ correspond to numerical results obtained in the framework of Eqs. (4)-(7). The theoretical curve corresponds to the Fig. 1 of Kharif et al. (2010).

interaction between the fundamental mode and its sidebands which dominates with a weak effect of the wind forcing and the dissipation. The second regime corresponding to the oscillatory evolution of the norm is dominated by the competition between wind forcing and dissipation. The nonlinear interaction between the fundamental mode and the sidebands is not the dominant mechanism. The magenta curve exhibits oscillations around an averaged value growing exponentially, whereas the yellow curve exhibits the same oscillations around a constant value. We can claim that the norm, $E_{n}$, presents globally exponential growth or asymptotical saturation corresponding to instability and stability, respectively. Herein, the stability can be interpreted in terms of asymptotic stability. The first case is said to be unstable, whereas the second case corresponds to a stable solution. In the latter case, we expect that the solution will remain close to the unperturbed solution. In other words, nonlinear interactions are affected by the non-conservative effects that are wind and dissipation, leading to a long time disappearance of these interactions.

Many numerical simulations have been run for various values of the parameters $\mathcal{A}$ and $\Omega$. Figure 6 shows a stability diagram which presents the comparison between the present numerical results and those of Kharif et al. (2010) obtained theoretically. The marginal curve corresponding to the fully nonlinear equations is very close to the theoretical marginal curve obtained within the framework the NLS equation. The region above the critical curve corresponds to stable cases, whereas the region beneath corresponds to unstable cases. Bars in Fig. 6 correspond to uncertainty on stability or instability. Numerical results obtained in our previous work (Kharif and Touboul, 2010) within the framework of Eqs. (4)-(7) are plotted for the sake of 
reference $(\circ)$. The way of introducing the damping effect into the kinematic boundary condition has little influence on the results, especially for young waves. The present numerical simulations demonstrate that the results derived by Kharif et al. (2010) within the framework of the NLS equation are correct in the context of the fully nonlinear equations.

\section{Conclusions}

This study was aimed at extending the work of Kharif et al. (2010) to the fully nonlinear case. Within the framework of the NLS equation the latter authors considered the modulational instability of Stokes wave trains suffering both effects of wind and dissipation. They found that the modulational instability depends on both frequency of the carrier wave and strength of the wind velocity and plotted the curve corresponding to marginal stability in the $(\mathcal{A}, \Omega)$-plane. To distinguish stable solutions from unstable solutions, we have introduced a norm based on the potential energy of the perturbations. A nonlinear stability diagram resulting from the numerical simulations of the fully nonlinear equation has been given in the $(\mathcal{A}, \Omega)$-plane which coincides with the linear stability analysis of Kharif et al. (2010). In the presence of wind, dissipation and modulational instability, it is found that wind energy goes to the subharmonic sideband, whereas dissipation lowers the amplitude of the fundamental mode of the wave train yielding to a permanent frequency-downshifting.

Acknowledgements. The authors would like to thank the referees who significantly helped improving the manuscript.

Edited by: E. Pelinovsky

Reviewed by: A. Babanin, L. Shemer, and another

anonymous referee

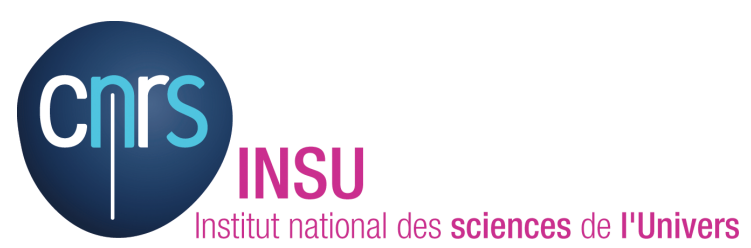

The publication of this article is financed by CNRS-INSU.

\section{References}

Banner, M. L. and and Tian, X.: On the determination of the onset of breaking for modulating surface gravity water waves, J. Fluid Mech., 367, 107-137, 1998.

Benjamin, T. B. and Feir, J. E.: The disintegration of wave trains on deep water, J. Fluid Mech., 27, 417-430, 1967.

Conte, S. D. and Miles, J. W.: On the numerical integration of the Orr-Sommerfeld equation, J. Soc. Ind. Appl. Maths., 7, 361-366, 1959.
Dias, F., Dyachenko, A. I., and Zakharov, V. E.: Theory of weakly damped free-surface flows: A new formulation based on potential flow solutions, Phys. Lett. A, 372, 1297-1302, 2008.

Dold, J. W. and Peregrine, D. H.: Water-wave modulation, Proc. 20th Intl. Conf. Coastal Eng., Taipei, 1, 163-175, 1986.

Dommermuth, D. G. and Yue, D. K. P.: A high-order spectral method for the study of nonlinear gravity waves, J. Fluid Mech., 184, 267-288, 1987.

Fermi, E., Pasta, J., and Ulam, S.: Studies of nonlinear problems, I, (reproduced in: Nonlinear Wave Motion, edited by: Newell, A. C., American Mathematical Society, Providence, R. I., 143-156, 1974), Los Alamos Report, LA-1940, 1955

Hara, T. and Mei, C. C.: Frequency downshift in narrowbanded surface waves under the influence of wind, J. Fluid Mech., 230, 429-477, 1991.

Joo, S. W., Messiter, A. F., and Schultz, W. W.: Evolution of weakly nonlinear water waves in the presence of viscosity and surfactant, J. Fluid Mech., 229, 135-158, 1991.

Kharif, C. and Pelinovsky, E.: Physical mechanisms of the rogue wave phenomenon, Eur. J. Mech. B-Fluid., 22(6), 603-633, 2003.

Kharif, C. and Ramamonjiarisoa, A.: Deep water gravity wave instabilities at large steepness, Phys. Fluids, 31, 1286-1288, 1988.

Kharif, C. and Touboul, J.: Under which conditions the BenjaminFeir instabilty may spawn a rogue wave: a fully nonlinear approach, Eur. Phys. J., Special Topics, 185, 159-168, 2010.

Kharif, C., Pelinovsky, E., and Slunyaev, A.: Rogue waves in the ocean, Springer, ISBN:978-3-540-88418-7, 216 pp., 89 illus., 2009.

Kharif, C., Kraenkel, R., Manna, M., and Thomas, R.: The modulational instability in deep water under the action of wind and dissipation, J. Fluid Mech., 664, 138-149, 2010.

Lamb, H.: Hydrodynamics, 6th edn., Cambridge University Press, 1932.

Lighthill, M. J.: Contributions to the theory of waves in non-linear dispersive systems, J. Inst. Math. Appl., 1, 269-306, 1965.

Longuet-Higgins, M. S.: Bifurcation in gravity waves, J. Fluid Mech., 151, 457-475, 1985.

Longuet-Higgins, M. S.: Theory of weakly damped Stokes waves: a new formulation and its physical interpretation, J. Fluid Mech., 235, 319-324, 1992.

Lundgren, T. S.: A free Surface Vortex Method with weak viscous effects, in: Mathematical Aspects of Vortex Dynamics, edited by: Caflisch, R. E., SIAM Proceedings, Ch. 6, 68-79, 1989.

Miles, J. W.: On the generation of surface waves by shear flow, J. Fluid Mech., 3, 185-204, 1957.

Ruvinsky, K. D., Feldstein, F. I., and Freidman, G. I.: Numerical simulations of the quasi-stationary stage of ripple excitation by steep gravity-capillary waves, J. Fluid Mech., 230, 339-353, 1991.

Segur, H., Henderson, D., Carter, J., Hammack, J., Li, C. M., Pheiff, D., and Socha, K.: Stabilizing the Benfamin-Feir instability, J. Fluid Mech., 539, 229-271, 2005a.

Segur, H., Henderson, D. M., and Hammack, J. L.: Can the Benjamin-Feir instability instability spawn a rogue wave?, Proc. 14th "Aha Huliko" a Hawaiian winter worshop, 43-57, 2005 b.

Skandrani, C., Kharif, C., and Poitevin, J.: Nonlinear evolution of water surface waves: the frequency down-shift phenomenon, 
Contemp. Math., 200, 157-171, 1996.

Shemer, L.: On Benjamin-Feir instability and evolution of a nonlinear wave with finite-amplitude sidebands, Nat. Hazards Earth Syst. Sci., in press, 2010.

Stokes, G. G.: On the theory of oscillatory waves, Transactions of the Cambridge Philosophical Society, 8, 441-455, 1847.

Touboul, J.: On the influence of wind on extreme wave events, Nat. Hazards Earth Syst. Sci., 7, 123-128, doi:10.5194/nhess-7-1232007, 2007.

Touboul, J. and Kharif, C.: On the interaction of wind and extreme gravity waves due to modulational instability, Phys. Fluids, 18, 108103, doi:10.1063/1.2374845, 2006.

Touboul, J., Giovanangeli, J. P., Kharif, C., and Pelinovsky, E.: Freak Waves under the Action of Wind: Experiments and Simulations, Eur. J. Mech. B. Fluid., 25, 662-676, 2006.

Touboul, J., Kharif, C., Pelinovsky, E., and Giovanangeli, J.-P.: On the interaction of wind and steep gravity wave groups using Miles' and Jeffreys' mechanisms, Nonlin. Processes Geophys., 15, 1023-1031, doi:10.5194/npg-15-1023-2008, 2008.
Trulsen, K. and Dysthe, K. B.: Frequency down-shift through self modulation, in: Proc. Water Wave Kinematics, edited by: Torum, A. and Gudmestad, O. T., Kluwer Academic Publishers, Series E, 178, 561-572, 1990.

Trulsen, K. and Dysthe, K. B.: Action of windstress and breaking on the evolution of a wavetrain, in: Breaking Waves, edited by Banner, M. L. and Grimshaw, R. H. J., Springer Verlag, 243-249, 1992.

West, B. J., Brueckner, K. A., Janda, R. S., Milder, M., and Milton, M.: A new numerical method for surface hydrodynamics, J. Geophys. Res., 92, 11803-11824, 1987.

Whitham, G. B.: Linear and nonlinear waves, Wiley-Interscience, New-York, 636 pp., 1974.

Wu, G., Liu, Y., and Yue, D. K. P.: A note on stabilizing the Benjamin-Feir instability, J. Fluid Mech., 556, 45-54, 2006.

Zakharov, V. E.: Stability of periodic waves of finite amplitude on the surface of a deep fluid, J. Appl. Mech. Tech. Phy., 9, 190194, 1968. 